\title{
Selbstverwaltung der Justiz: Ein Modell auch für Deutschland
}

\section{Einleitung und Gang der Darstellung}

Über Selbstverwaltung der Justiz oder besser die Selbstständigkeit und Autonomie in der Justiz wird in Deutschland seit den fünfziger Jahren diskutiert. Der 40. Deutsche Juristentag 1953 und der 63. Deutsche Juristentag 2002 haben sich mit der Thematik befasst, der Justiz die Stellung zu verschaffen, die ihr nach dem Gewaltenteilungsprinzip des Grundgesetzes und nach der in den Artikeln 92 ff. GG vorgesehenen Gerichtsorganisation zugewiesen ist. Die dort erhobenen verfassungsrechtlichen Bedenken wurden jedoch als unüberwindbar angesehen, einen politischen Willen zu Verfassungsänderungen zum Status der Justiz gab es nicht. Gerade wegen der - auch im internationalen Vergleich - anerkannt hohen Leistungsfähigkeit der Justiz wurde ein Reformbedarf für die Justizverfassung nicht gesehen. Der Hinweis auf europäische Standards und die Umsetzung des Selbstverwaltungsgedankens in europäischen Nachbarstaaten wurde mit pauschaler Kritik an der Politisierung kooptierender, sich demokratischer Kontrolle und exekutiver Steuerung entziehender Justizsysteme abgetan (»Wollt ihr Verhältnisse wie in Italien oder Spanien?«). Eine umfassende vergleichende Untersuchung der so diskriminierten ausländischen Systeme hat es bis heute nicht gegeben. Die Diskussion zu strukturellen Änderungen ist im Wesentlichen in den Gremien der Richterverbände geführt worden, findet aber erst seit dem vergangenen Jahr in der Kollegenschaft selbst, bei Rechtspolitikern und in der öffentlichen Wahrnehmung wieder breiteres Interesse.

Der Deutsche Richterbund hat die Ausgangsfrage nach dem »Ob« der Selbstverwaltung in Deutschland schon klar beantwortet. Auf seiner Bundesvertreterversammlung am 27.04.2007 hat der DRB die Selbstverwaltung gefordert und sich zu ihrer Umsetzung in einem Eckpunktepapier für ein konkretes so genanntes Zwei-Säulen-Modell entschieden. Zum »Wie« erwarten wir offene politische Diskussionen unter Einbeziehung unseres Modells und natürlich Anregungen auch aus dieser Tagung.

Ich möchte Ihnen aus einer Beschreibung der Verfassung und des Zustandes der Justiz Änderungsbedarf begründen, unser Selbstverwaltungsmodell kurz vorstellen und schließlich unsere Erwartungen an die Umsetzung des Gedankens der Selbstverwaltung in Deutschland formulieren. Der vorgegebene Zeitrahmen zwingt zu verkürzenden Darstellungen. ${ }^{1}$

\section{Status der Justiz}

Das Grundgesetz für die Bundesrepublik Deutschland sieht die Justiz als Institution vor und vertraut die Rechtsprechung den Gerichten an. Die Verfassung zeichnet jedoch kein geschlossenes Richterbild, dieses erschließt sich vielmehr aus dem Zusam-

1 Ich verweise für nähere Informationen auf die Homepage des DRB. 
menhang der Artikel 20, 28, 92, 97 und 103 GG. Aus dem Rechtsstaatsprinzip gemäß den Artikeln 20 und 28 GG ergeben sich die Bindung an Gesetz und Recht, die Unabhängigkeit der Richter, die Gewaltenteilung und die Gewährleistung eines fairen Verfahrens. Wir haben in Deutschland einen hohen Standard persönlicher Unabhängigkeit der Richter und - in der Praxis - auch der Staatsanwälte. Die Verwaltung der Gerichte und Staatsanwaltschaften erfolgt durch den Bundesjustizminister und die Justizminister der 16 Bundesländer. Personal- und Haushaltsverantwortung liegen also bei der Exekutive. Das Bundesverfassungsgericht besitzt eine eigene Personal- und Budgethoheit, es verwaltet sich selbst.

\section{Praktische Lage der Justiz}

\section{1. Überlastung durch steigende Verfahren und gleichzeitigen Personalmangel}

Die Justiz ist flächendeckend überlastet, der Nachweis ist durch PEBB§Y, eine von den Landesjustizverwaltungen in Auftrag gegebene externe betriebswirtschaftliche Organisationsuntersuchung, geführt. Während die Zahl der Rechtsanwälte auf heute ca. 149.000 zugenommen hat, ist die Zahl der Richter (ca. 20.400) und der Staatsanwälte (ca. 5.000) trotz steigender Zahl der Verfahren und weiterer Verrechtlichung der Gesellschaft in den letzten 10 Jahren ständig zurück gegangen. Im Jahr 1995 gab es noch 22.100 Richter und 5.400 Staatsanwälte. Den Justizministern, die unter Kabinetts- und Parteizwängen stehen und Sparvorgaben umzusetzen haben, gelingt es also trotz ihres Engagements nicht, ausreichende Mittel zu beschaffen und den in der Verfassung verankerten Justizgewährungsanspruch der Bürger umfassend zu sichern. Selbst bei offensichtlichem Fehlbedarf sehen viele Justizminister ihre Rolle darin, Kürzungskonzepte zu verteidigen, statt Haushaltsdiskussionen zu nutzen, um sich berechtigte Forderungen der Justiz zu eigen zu machen. Eine Grundsatzdiskussion über die Wertigkeit und gesellschaftspolitische Stabilisierungsfunktion findet nicht in ausreichendem Maße statt. Exekutive und Jurisdiktion sind in dieser Konstellation verteilungspolitische Gegner.

\section{Folgen der unzureichenden Personalausstattung}

Die Auswirkungen des Personalnotstandes sind offensichtlich:

- längere Verfahrenszeiten im Zivilrecht mit Gefährdung des noch erfolgreichen und angesehenen Rechtsstandorts Deutschland,

- längere Verfahrenszeiten bei Familiengerichten mit sozialen Folgelasten,

- längere Verfahrenszeiten in den Fachgerichtsbarkeiten mit erheblichen sozialen und finanziellen Auswirkungen,

- keine zeitnahe Reaktion im Jugendrecht,

- Haftentlassungen wegen Verfahrensverzögerungen, um nur einige Beispiele zu nennen.

Zwei weitere Folgen möchte ich zusätzlich ansprechen, weil sie das Grundverständnis unseres Berufes in besonderer Weise betreffen: Wegen fehlender Ressourcen nimmt im Bereich des Strafrechts der Druck zu informellen Erledigungen zu. Dies gilt 
im besonderen Maße für Absprachen im Strafprozess. Die unverzichtbare Stabilisierungsfunktion des Strafrechts für unser Gemeinwesen wird aufs Spiel gesetzt. Die Praxis entfernt sich zunehmend vom Legalitätsprinzip und trifft Opportunitätsentscheidungen, die systemwidrig sind und das Gerechtigkeitspostulat gefährden. Die Arbeit von Richtern und Staatsanwälten wird nach betriebswirtschaftlichen Parametern gemessen. Steuerungsmodelle aus der Verwaltung werden auf die Justiz übertragen, ohne dass den Besonderheiten der richterlichen Unabhängigkeit und der besonderen Stellung der Staatsanwaltschaft Rechnung getragen wird.

Die Quantität der Arbeit, nicht ihre Qualität ist entscheidendes Anknüpfungsmerkmal für Personalzuweisungen, aber auch für konkrete Personalentscheidungen wie Beförderungen. Personalentscheidungen, von der Einstellung bis zur Beförderung in Spitzenämter, werden in vielen Ländern von der Exekutive nach politischen Proporzüberlegungen als Steuerungsinstrumente verstanden. Sie erzeugen zumindest den bösen Anschein der Einflussnahme auch auf die persönliche Unabhängigkeit der Richter und Staatsanwälte.

\section{Stellung des deutschen Justizsystems im europäischen Vergleich}

Wir werden hierzu mit Beiträgen aus erster Hand informiert. Deshalb an dieser Stelle nur einige Feststellungen in Kurzform zur Darstellung der Diskussion in Deutschland: Die überwiegende Mehrheit der Mitglieder der Europäischen Union hat sich für eine selbstverwaltete Justiz entschieden. Die deutsche Justizstruktur widerspricht offensichtlich den Empfehlungen des Europarates, wonach die für die Auswahl und Laufbahn der Richter zuständige Stelle von der Exekutive unabhängig sein muss. Die Staatsanwaltschaft wird entgegen ihrem nach dem Legalitätsprinzip nur Recht und Gesetz verpflichteten gesetzlichen Auftrag durch das europäischen Vorgaben widersprechende ministerielle Weisungsrecht in den Verdacht politisch beeinflusster Entscheidungen gebracht. In internationalen Gremien kommen wir uns oft wie ertappte Sünder vor, die zwar wegen ihrer Rechtskultur und ihrer überzeugenden Arbeit hohes Ansehen genießen, mit den Justizverwaltungsstrukturen den Grundsatz der Gewaltenteilung aber nicht strikt umsetzen.

\section{Stand der politischen Diskussion}

In das - übrigens parteiübergreifende - Abwehrbollwerk gegen eine Selbstverwaltung der Justiz ist Bewegung gekommen: Das Bundesjustizministerium, das als Exekutivorgan in diesem Gremium selbstverwalteter Justizen ohnehin nur Beobachterstatus hat, lässt sich auf der Versammlung des Europäischen Netzwerks der Justizverwaltungsräte (ENCJ) seit diesem Jahr begleitend und in Absprache mit den anderen deutschen Richterverbänden durch den DRB vertreten. Der Koalitionsvertrag der schwarzgrünen Regierung in Hamburg sieht die Verpflichtung vor, einen offenen Diskussionsprozess zu Möglichkeiten der Verbesserung der Selbstständigkeit der Justiz zu führen. 
Sie haben hierzu Justizsenator Dr. Steffen gehört. Er will 2010 hiermit die Justizministerkonferenz befassen; die Länder werden sich dann insgesamt positionieren müssen.

In Schleswig-Holstein hat Justizminister Döring vor wenigen Wochen angekündigt, bis 2010 die gesamte Haushaltsverantwortung den Gerichten zu übertragen. Er hat Kommissionen einberufen, die Vorschläge für diese neuen Verwaltungsstrukturen entwickeln sollen. Die für uns unverzichtbare Einbeziehung der Staatsanwaltschaft sieht seine Planung bisher allerdings nicht vor. Mit den Anwaltsorganisationen stehen wir in einem offenen Meinungsaustausch, auch zu Überlegungen, wie die Anwaltschaft in den Gremien einer selbstverwalteten Justiz mitwirken könnte.

In zahlreichen Veranstaltungen wird das Thema interessiert aufgegriffen. Bei der Berichterstattung über - vermeintliche - Missstände in der Justiz werden regelmäßig die beschriebenen Ausstattungsdefizite in den Zusammenhang einer Diskussion über die Abhängigkeit der Justiz von Entscheidungen der Exekutive gestellt. Gefragt wird, ob es einer selbstverwalteten Justiz besser gelingen könnte, die Voraussetzungen zu schaffen, dass die berechtigten Erwartungen der Bürger an ein funktionierendes Rechtssystem erfüllt werden. Das Interesse am Thema Selbstverwaltung ist also untrennbar und legitimerweise mit den Erwartungen an Verbesserungen verbunden. Was kann die Justiz unter eigener Regie nun besser erledigen?

a. Qualitätsverbesserung durch Sachnähe der Entscheidungsträger

b. Unmittelbare Wahrnehmung des Bedarfs an Sachmitteln und Personal

c. Vermittlung des Personalbedarfs in einem transparenten Diskussionsprozess mit klaren politischen Forderungszuweisungen

d. Personalauswahl, Ernennungen, Versetzungen, Einstellungs- und Anforderungsprofile ohne politische Einflussnahme und politisches Proporzdenken

e. Offene interne Debatte über Qualität und richterliche Ethik

f. Höhere Akzeptanz für Beurteilungen und Beförderungen

g. Authentische Öffentlichkeitsarbeit

h. Sachnähere und damit besser akzeptierte Dienstaufsicht

i. Bedarfsgerechte Aus- und Fortbildung

\section{Das Zwei-Säulen-Modell des Deutschen Richterbundes}

Wie stellen wir uns ein Selbstverwaltungsmodell für die deutsche Justiz vor? Der DRB hat in Eckpunkten ein Modell als Grundlage für die weitere Diskussion beschlossen, das die Justiz aus der Steuerung durch die Exekutive löst, sie zugleich aber stärker und unmittelbarer gegenüber dem Parlament verantwortlich macht. Die Selbstverwaltungsorgane sollen höchstmöglich demokratisch legitimiert sein.

- Das Modell sieht vor, dass an die Stelle des Justizministers ein Justizverwaltungsrat aus Richtern und Staatsanwälten tritt (erste Säule).

- Seine Mitglieder sollen von einem Justizwahlausschuss gewählt werden, dem mehrheitlich Landtagsabgeordnete und daneben gewählte Richter aus allen Gerichtsbarkeiten sowie Staatsanwälte angehören (zweite Säule).

- Zu den Aufgaben des Justizverwaltungsrates gehören insbesondere alle Personalentscheidungen und die Dienstaufsicht in der Justiz. Er stellt - besonders wichtig - 
das Gesamtbudget der Justiz auf, vertritt es direkt gegenüber dem Finanzminister und - bei Nichteinigung - gegenüber dem Parlament. Kontroverse Personalentscheidungen werden an den Justizwahlausschuss abgegeben. Verwaltungsaufgaben, die gegenwärtig dezentral von den Gerichten und Staatsanwaltschaften selbst erledigt werden, sollen dort verbleiben.

- Die Staatsanwaltschaft soll an der Selbstverwaltung der Justiz teilnehmen. Dies entspricht ihrer Stellung innerhalb der Dritten Gewalt als eine dem Richteramt ähnliche. Sie ist ein der Dritten Gewalt gleich- und zugeordnetes Organ der Rechtspflege und erfüllt im Strafrecht gemeinsam mit den Gerichten die Aufgabe der Justizgewährung.

- Bei den Justizministerien verbleiben die Zuständigkeiten für die Erarbeitung von Gesetzen, die Juristenausbildung, die Notaraufsicht, die Strafvollstreckung und die Gnadensachen.

- Die Mitwirkungsgremien in Gerichten und Staatsanwaltschaften bleiben erhalten.

\section{Realisierungschancen}

In den vergangenen Monaten hat das Interesse am Thema Selbstverwaltung der Justiz deutlich zugenommen. Die Forderung nach Selbstverwaltung ist als zentrales Anliegen des DRB und der anderen Berufsverbände bekannt. Wer sich mit unserer Auffassung näher auseinandersetzt, kann sich nun vorstellen, dass von einem gewählten Justizverwaltungsrat selbst direkt gegenüber dem Parlament erhobene Haushaltsanforderungen, die für den Bürger transparente Wirkweisen der finanziellen Ausstattung der Justiz aufzeigen, eine offene und öffentliche Diskussion über den Stellenwert der Justiz auslösen werden. Die Politik reagiert dort bereits, wo, wie in Hamburg und Schleswig-Holstein, die Situation der Justiz leidenschaftslos, ehrlich und ohne den schlichten Reflex der Sicherung der Macht des eigenen Amtes bewertet wird. Die politische Diskussion ist damit unumkehrbar eröffnet. Sie wird Chancen bieten, in den Ländern aus den Erfahrungen mit ihren schon bestehenden Mitwirkungsmodellen heraus verschiedene Wege zu einem richtigen Ziel zu finden. Immerhin haben wir als eines der letzten Länder, das in Europa um die Selbstverwaltung seiner Justiz ringt, auch die Möglichkeit, andere Systeme zu bewerten und aus Erfahrungen in anderen Staaten zu lernen. 